\title{
Using time-to-contact information to assess potential collision modulates both visual and temporal prediction networks
}

\author{
Jennifer T. Coull ${ }^{1, *}$, Franck Vidal ${ }^{1}$, Ceydric Goulon ${ }^{2}$, Bruno Nazarian ${ }^{3}$ and \\ Cathy Craig ${ }^{2,4}$ \\ 1. Laboratoire de Neurobiologie de la Cognition, Université Aix-Marseille \& CNRS, Marseille, France \\ 2. Institut des Sciences du Mouvement, Université Aix-Marseille, Faculté des Sciences du Sport, Marseille, France \\ 3. Centre IRMf de Marseille, CHU Timone, Marseille, France \\ 4. School of Psychology, Queen's University Belfast, Northern Ireland, UK
}

Edited by: $\quad$ Anna C. Nobre, University of Oxford, UK

Reviewed by: Ricarda I. Schubotz, Max Planck Institute of Human Cognitive and Brain Sciences, Germany

Salvatore M. Aglioti, Universita degli studi di Roma, Italy

Anna C. Nobre, University of Oxford, UK

\begin{abstract}
Accurate estimates of the time-to-contact (TTC) of approaching objects are crucial for survival. We used an ecologically valid driving simulation to compare and contrast the neural substrates of egocentric (head-on approach) and allocentric (lateral approach) TTC tasks in a fully factorial, event-related fMRI design. Compared to colour control tasks, both egocentric and allocentric TTC tasks activated left ventral premotor cortex/frontal operculum and inferior parietal cortex, the same areas that have previously been implicated in temporal attentional orienting. Despite differences in visual and cognitive demands, both TTC and temporal orienting paradigms encourage the use of temporally predictive information to guide behaviour, suggesting these areas may form a core network for temporal prediction. We also demonstrated that the temporal derivative of the perceptual index tau (tau-dot) held predictive value for making collision judgements and varied inversely with activity in primary visual cortex (V1). Specifically, V1 activity increased with the increasing likelihood of reporting a collision, suggesting top-down attentional modulation of early visual processing areas as a function of subjective collision. Finally, egocentric viewpoints provoked a response bias for reporting collisions, rather than no-collisions, reflecting increased caution for head-on approaches. Associated increases in SMA activity suggest motor preparation mechanisms were engaged, despite the perceptual nature of the task.
\end{abstract}

Keywords: collision, timing, prediction, attention, fMRI, visual, parietal, premotor

\section{INTRODUCTION}

Accurate estimates of the time-to-contact (TTC) of approaching objects are used effortlessly in our everyday lives to guide future motor behaviour. Examples include catching a ball, crossing a busy road, or braking to avoid an obstacle. Lee $(1976,1998)$ has developed a theory of TTC in which a low-level perceptual index, tau, allows direct estimation of the rate of closure of the gap between the observer and an object, thus enabling the prospective control of movement. For visual stimuli, tau is perceived directly from the changing visual properties of the stimulus, more specifically the ratio between image size and its rate of change. Although this ratio specifies the time remaining before contact (i.e. TTC), one oft-criticised feature is that it does not allow for

*Correspondence: Jennifer T. Coull, Laboratoire de Neurobiologie de la Cognition, Université Aix-Marseille \& CNRS, Pôle 3C, 3 place Victor-Hugo, 13331 Marseille cedex 3 , France. e-mail: jennifer.coull@univ-provence.fr

Received: 17 June 2008; paper pending published: 07 July 2008; accepted: 05 September 2008; published online: 13 September 2008.

Citation: Front. Hum. Neurosci. (2008) 2: 10. doi: 10.3389/neuro.09.010.2008

Copyright (C) 2008 Coull, Vidal, Goulon, Nazarian and Craig. This is an openaccess article subject to an exclusive license agreement between the authors and the Frontiers Research Foundation, which permits unrestricted use, distribution, and reproduction in any medium, provided the original authors and source are credited. changes in velocity. The first-order temporal derivative of tau, tau-dot, does however, and it can be used to estimate the sufficiency of braking when avoiding collisions (e.g. "am I braking hard enough to avoid running into this obstacle?"). Specifically, tau-dot is used to determine whether the current rate of gapclosure (i.e. braking speed) would result in collision or not. The prospective nature of this information thus allows subjects to modify their current behaviour, if necessary, in order to avoid collision (e.g. brake harder). Although it was originally suggested (Lee, 1976) that subjects control braking by actively maintaining a constant tau-dot value during the approach, the evidence for a constant tau-dot braking strategy is conflicting and ultimately unconvincing (e.g. Bootsma and Craig, 2003; Kim et al., 1993; Rock and Harris, 2006; Yilmaz and Warren, 1995). Instead, results shows that tau-dot information is perceived passively from changing visual properties and informs about the likelihood of collision, which then allows for dynamic adjustments in braking behaviour so as to avoid collision (Bootsma and Craig, 2003; Yilmaz and Warren, 1995).

Bootsma and Craig (2003) demonstrated that tau-dot can be used to determine braking sufficiency not only for scenarios in which the observer is on a collision course with an object (head-on approach or "egocentric" viewpoint), but also 
for scenarios in which objects in the external environment are on a collision course with one another (transversal approach or "allocentric" viewpoint). Using fMRI, Assmus et al. (2003, 2005) demonstrated left inferior parietal cortex activations for allocentric TTC judgements while Field and Wann (2005) reported left sensori-motor cortex activity for egocentric ones. Although Field and Wann (2005) also reported activity related to allocentric TTC tasks, their control task was poorly-matched to the allocentric task: stimuli in both egocentric and control tasks moved radially whereas allocentric stimuli moved laterally. The results were therefore rather ambiguous and furthermore the design precluded direct comparison of allocentric to egocentric tasks due to the visual differences in stimulus display. The first aim of the present study was to use ecologically valid stimuli to directly compare differences and identify commonalities in the neural instantiation of egocentric and allocentric TTC tasks, whilst simultaneously controlling for differences in sensori-motor stimulus properties through the use of a fully factorial design. In addition, unlike previous TTC studies, we used an event-related design in order to correlate differing values of tau-dot to changes in brain activity.

The context for this study stems from previous investigations into the neural basis of temporal orienting (Coull and Nobre, 1998; Nobre, 2001). In this task, subjects use informative cues to direct attentional resources prospectively towards a predictable moment in time in order to speed target detection. There is a clear conceptual link between the use of temporal orienting cues to increase response speed and of TTC information to enable the prospective control of movement. However, while temporal predictability is established explicitly by attentional cues in the orienting task, spatio-temporal predictability is conveyed implicitly by object motion in the TTC task. In addition, the current TTC task requires delayed perceptual judgements rather than speeded motor responses. Any neuroanatomical overlap in the areas activated by the orienting (temporal, explicit, motor) or TTC task (spatio-temporal, implicit, perceptual) would therefore reflect a generic network for temporal prediction. Thus, the second aim of the present study was to identify the neural substrates of the cognitive process that is common to these two different paradigms (i.e. temporal prediction) by pinpointing brain regions whose activity is common to the two paradigms.

\section{MATERIALS AND METHODS SUBJECTS}

Twelve healthy, right-handed male volunteers (mean age $=$ 23.5 years, range $=21-26$ years) participated in the study. The experimental protocol was approved by the local ethics committee, and written informed consent was obtained prior to the study.

\section{COGNITIVE TASKS}

One of our aims was to use an ecologically-valid stimulus display. To this end, subjects viewed a short (2-3.5 s) animated simulation (Supplementary Material 1) of a car driving towards a wall (Figure 1). Virtual reality software was used to create animated simulations of the car's trajectory, in a 3-dimensional space, using the distance and movement parameters defined in Supplementary Material 2. Throughout the animation, the car decelerated at a constant rate but the animation ended before the car came to a complete stop. The colour of the car changed gradually throughout its trajectory, while the colour of the wall remained constant. The animation was shown either from the driver's point of view (egocentric, Figure 1A) or from a bird's eye view (allocentric, Figure 1B) and the same animations were used for both experimental (TTC) and control (COL) tasks. This resulted in a $2 \times 2$ factorial design yielding four conditions: TTCego, TTCallo, COLego, COLallo.

In the TTC condition, the subject's task was to judge whether the car would have come into contact with the wall had the animation continued. In the COL condition, the subject's task was to judge whether the colour of the car and the wall had ever matched. The reason for having a dynamic, rather than static, car-colour property was to encourage subjects to attend to colour throughout the duration of the animation rather than simply estimating car-colour at stimulus-onset and then shifting attention to some other feature of the stimulus-display for the remainder of the animation. In this way, sustained attention requirements were approximately equated across both TTC and COL tasks. Moreover, requiring subjects to compare the temporally dynamic colour of the car with the colour of the wall roughly equated the visual comparison processes required in the TTC task, in which subjects had to compare the temporally dynamic spatial position of the car with the position of the wall. Therefore, the TTC task was the only one to require the use of prospective spatio-temporal information, but both tasks were matched for other, more general, sensori-motor and attentional processes.

The starting distance of the car from the wall (62.505 or $57.69 \mathrm{~m})$, the car's initial speed $\left(29.70\right.$ or $\left.30.30 \mathrm{~ms}^{-1}\right)$ and rate of deceleration $\left(7.20 \mathrm{~ms}^{-2}, 7.80 \mathrm{~ms}^{-2}\right)$, and the distance from the wall at which the animation ended ( 6 or $12 \mathrm{~m}$ ) varied pseudo-randomly from trial to trial. From these values, we calculated 16 different values of tau-dot $(\dot{\tau})$ at animation-offset (Supplementary Material 2) using the equation:

$\dot{\tau}=\frac{X \ddot{X}}{\dot{X}^{2}}-1 \quad($ where $X=$ distance, $\dot{X}=$ speed, $\ddot{X}=$ acceleration $)$

Tau-dot is the temporal derivative of tau (i.e. the rate of change of tau) and takes rates of acceleration/deceleration into account. While tau can be used to estimate time remaining before contact (the time between animation offset and the theoretical moment of contact ranged from 533 to $1329 \mathrm{~ms}$ in this experiment), tau-dot can be used to detect the sufficiency of braking thus allowing the current course of action to be modified so as to avoid a collision (Bootsma and Craig, 2003; Kim et al., 1993; Rock and Harris, 2006; Yilmaz and Warren, 1995). Mathematically it has been shown that a tau-dot value of -0.5 represents the cut-off value between a collision and no-collision scenario. The stimulus parameters used in this experiment gave rise to eight scenarios in which there would have been contact between the car and wall had the animation continued ("contact trial", tau-dot $<-0.5$ ), and eight in which there would have been no contact ("no-contact trial", tau-dot $\geq-0.5$ ). Each of these 16 scenarios was used (in pseudo-random order) for each of the four conditions (TTCego, TTCallo, COLego, COLallo), resulting in 64 trials per run. Subjects performed four runs of trials. Note that although the same stimuli were used for all four conditions (in order to match visual input) the tau-dot value of a particular scenario was incidental to task requirements in the COL condition.

The dynamic car-colour and static wall-colour also varied pseudo-randomly from trial to trial, in 16 different colourcombinations. The car-colour changed according to a sliding 


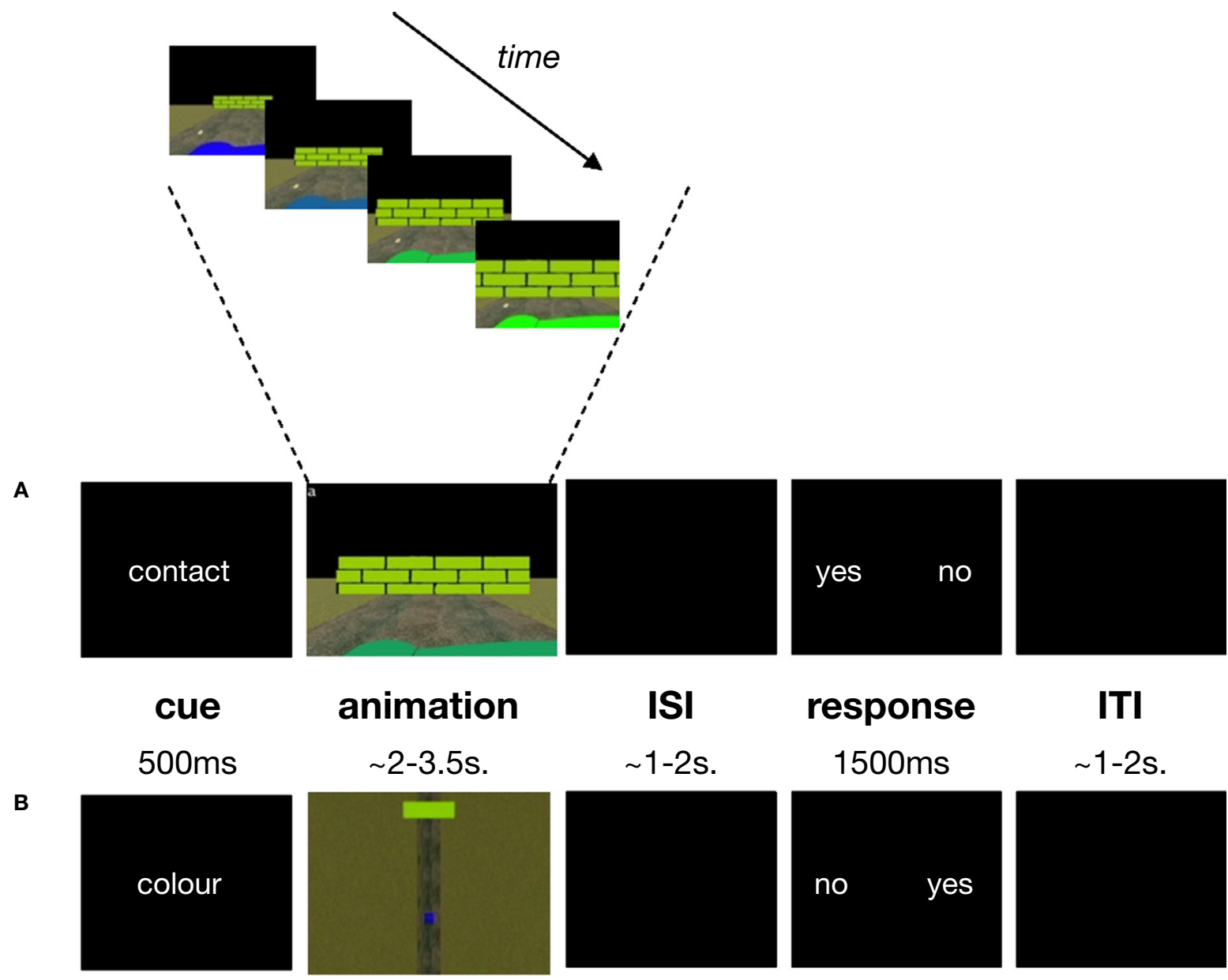

Figure 1 | Task structure and timing. (A) contact egocentric trial (B) colour allocentric trial. A briefly-presented cue ("contact" or "colour") instructed subjects to make time-to-contact (TTC) or colour judgements for a forthcoming animation. During the animation subjects saw a car (the dark green foreground object in panel $(\boldsymbol{A})$; the blue lower field object in panel $(\boldsymbol{B})$ ) approaching a wall (the light green object in panels $(\boldsymbol{A})$ and $(\boldsymbol{B})$ ) either from the driver's point of view (egocentric condition $(\boldsymbol{A})$ ) or from a bird's eye view (allocentric condition $(\boldsymbol{B})$ ). The TTC task was to estimate potential contact between the car and wall while the colour task was to detect a possible colour-match between the car and wall. Subjects responded to "yes" or "no" response options presented on the screen, whose positions varied from trial to trial. Subjects made an index- or middle-finger right-handed button-press corresponding to whether their contact or colour-match judgement (yes/no) appeared on the left or right of the screen, respectively. The colour of the car changed gradually throughout its trajectory, while the colour of the wall remained constant (top panel). Exactly the same animations were used for the TTC and colour tasks. ISI = inter-stimulus interval; ITI = inter-trial interval.

colour-scale in steps of $300 \mathrm{~ms}$. In eight of these colourcombinations, the colour of the wall matched the colour of the car during one of these 300-ms steps (the match occurred pseudo-randomly within the last $60-90 \%$ of stimulus presentation in order to encourage subjects to maintain attention to colour throughout). In the eight remaining colour-combinations, the car-colour never matched the wall-colour. These 16 colourcombinations were paired pseudo-randomly with the 16 contact scenarios described above, such that colour-match/no-colourmatch trials were counterbalanced with respect to contact/nocontact trials.

An experimental trial started with the presentation (500 ms) of an informative cue, either the word "contact" or "colour", which instructed subjects to make either contact or colour judgements on the forthcoming animated simulation. This was immediately followed by the 2-3.5 s animation described above. Finally, following a pseudo-random delay (1-2 s), a responsesignal was presented for $1500 \mathrm{~ms}$. During this response period, subjects pressed one of two response buttons corresponding to their contact or colour judgement ("yes" for a contact/colourmatch decision or "no" for a no-contact/no-colour-match decision). Due to the neuroanatomical overlap in areas related to motor preparation with those of timing [e.g. SMA (Coull et al., 2004; Lee et al., 1999)] and temporal expectation [e.g. left parietal cortex (Coull and Nobre, 1998; Rushworth et al., 2003)], we aimed to minimise motor preparation confounds as far as possible. Therefore, the manual response (index/middle finger) associated with each kind of judgement (yes/no) could change on a trial-by-trial basis. Specifically, the words "yes" or "no" could appear on either the left or right side of the screen (see Figure 1). If the word corresponding to the subject's judgement appeared on the left, the subject responded with the index finger of their right hand, and if it appeared on the right they responded with their middle finger. In this way, even if subjects made their decision (on a cognitive level) during presentation of the animation, they could not begin to prepare the appropriate response (at the motor level) until presentation of the response signal, thus dissociating processes of temporal expectation 
and motor preparation. Index-/middle-finger responses were counter-balanced for yes/no judgements across all trials. Intertrial intervals varied pseudo-randomly from 1-2 s. All subjects performed a training session consisting of 16 trials per condition, prior to fMRI scanning.

\section{fMRI SCANNING}

Scans were acquired using a 3-Tesla Bruker Medspec 30/80 Advance whole body MRI system, equipped with a head coil Echo-planar imaging (EPI) was used to obtain $\mathrm{T}^{*}$-weighted fMRI images in the axial plan, using an interleaved slice acquisition sequence. The acquired image volume consisted of $30 \times 4 \mathrm{~mm}$ transverse slices $(3 \times 3 \times 4 \mathrm{~mm}$ resolution), with an inter-scan interval (TR) of $1.91 \mathrm{~s}$. The size of this image volume allowed us to scan the entire cerebral cortex and the majority of the cerebellum (the ventral third of the cerebellum was not scanned). Four scanning runs (240 image volumes, approximately $8 \mathrm{~min}$, per run) were acquired for each subject. Given the trial length of $\sim 7.5 \mathrm{~s}$, the four conditions were presented in pseudo-random permuted order throughout each run in order to optimise signal strength (Josephs and Henson, 1999). Subjects performed a total of 64 trials per condition. Pseudo-randomisation of intertrial and inter-event intervals ensured random sub-sampling of the brain volume relative to each of the event-types. A structural MRI was also acquired (using a standard T1-weighted scanning sequence, $1.5 \mathrm{~mm}^{3}$ resolution) to allow anatomically specific localisation of significant areas of brain activation.

\section{DATA ANALYSIS}

\section{Behavioural data}

Accuracy (percentage of trials correct) and reaction times (RTs) were recorded during the fMRI session. Mean values were compared using a repeated-measures ANOVA, with task (TTC vs. $\mathrm{COL}$ ) and viewpoint (egocentric vs. allocentric) as the two factors of interest. Using tau-dot values $<$ or $\geq-0.5$ to divide TTC trials into contact or no-contact trials respectively, we further categorised correct and incorrect responses as hits ("yes" decision on a contact trial), correct rejections ("no" decision on a nocontact trial), false alarms (FA) ("yes" decision on a no-contact trial) and misses ("no" decision on a contact trial). A similar categorisation was conducted on colour trials, except that match and no-match trials were initially identified on the basis of coincident car/wall colour values rather than tau-dot values. We then performed an analysis on these data using Signal Detection Theory in order to obtain estimates of sensitivity index $\left(d^{\prime}\right)$ and response bias $(\beta)$, using the formulae:

$d^{\prime}=Z($ hit $)-Z(\mathrm{FA})$

$\beta=\exp \left(-0.5 \times d^{\prime} \times(Z(\right.$ hit $)+Z(\mathrm{FA}))$

The sensitivity index $\left(d^{\prime}\right)$ indicates how well the subject correctly discriminated "yes" (e.g. contact) from "no" (e.g. no-contact) trials. The response bias $(\beta)$ indicates the extent to which subjects were more likely to respond "yes" or "no".

Finally, to verify how well the informational quantity tau-dot could predict subjects' performance in the TTC and colour conditions, the percentage of trials in which the subjects made a "contact" decision, for egocentric or allocentric viewpoints, were plotted separately against tau-dot values. A logistic regression analysis was used to calculate $R^{2}$ values that indexed how well the value of tau-dot (independent variable) predicted how often a subject would make a "contact" decision (dependent variable).
Logistic regression was used in favour of linear regression since our dependent variable was dichotomous (e.g. yes/no) rather than parametric. Given that tau-dot has already been demonstrated to predict the likelihood of ensuing collisions (Bootsma and Craig, 2003) it was predicted that the analysis would yield high $R^{2}$ values for performance in the TTC conditions and low $R^{2}$ values for performance in the colour conditions.

\section{fMRI data}

Image processing and analysis of fMRI data were conducted with SPM2 (http://www.fil.ion.ucl.ac.uk/spm/software/spm2; Friston et al., 1995a,b). All functional images for each subject were slice-time corrected to a slice acquired half-way through image acquisition (corresponding anatomically to a slice near the top of the brain) in order to correct for temporal differences (up to $2 \mathrm{~s}$ ) between slices acquired early, and those acquired late, in the image volume. These images were then realigned to correct for head movement between scans. Each structural MRI was co-registered to the corresponding mean realigned functional image, in order to put structural images into the functional brain space. All images were then spatially normalised into a standard spatial frame by matching each image to a standardised template from the Montreal Neurological Institute. Functional images were spatially smoothed to accommodate inter-subject differences in anatomy, using isotropic Gaussian kernels of $8 \mathrm{~mm}$.

Task-evoked neural responses were modelled as single events (stick function) that were time-locked to the onset of the animated simulation and then convolved with a canonical haemodynamic response function (hrf) (Friston et al., 1998). This was conducted separately for each experimental condition, resulting in four distinct regressors. Data were analysed for regionally specific changes in hrf amplitude as a function of experimental condition. A parametric modulation of TTC regressors by the ranked tau-dot value per trial was also included in the statistical model. Finally, in order to explain as much of the experimental variance as possible an additional regressor, time-locked to presentation of the response signal across all four conditions collectively, was also modelled.

Condition effects were estimated according to the general linear model at each voxel in brain space in each of the 12 subjects. Images were adjusted for both global intensity, using proportional scaling; and for low-frequency physiological drifts, using a highpass filter of $128 \mathrm{~s}$. Data were subject to a randomeffects analysis, which allowed inferences derived from this subject sample to be generalised to the population. At the first level of analysis, we performed 12 separate single-subject analyses. For each subject, we defined statistical parametric maps (SPMs) of the $t$ statistic (transformed into corresponding $Z$ values) for each of the following comparisons between regressors:

\section{Colour-related activity}

Regions associated with egocentric representations of colourprocessing were identified by the simple main effect of task in trials showing the driver's viewpoint [COLego-TTCego] while those associated with allocentric representations were identified by the complementary contrast in bird's-eye view trials [COLallo-TTCallo]. Regions common to both egocentric and allocentric representations of colour-processing were identified by inclusively masking the [COLallo-TTCallo] contrast with the [COLego-TTCego] contrast (thresholded at $p<0.01$ uncorrected for multiple comparisons). This "conjunction" analysis defines a fundamental colour-processing network, which is recruited for colour judgements whatever the viewpoint. 


\section{TTC-related activity}

Regions associated with egocentric representations of TTC were identified by the simple main effect of task for trials showing the driver's viewpoint [TTCego-COLego], while those associated with allocentric representations of TTC were identified by the complementary contrast in bird's-eye view trials [TTCalloCOLallo]. Regions common to both egocentric and allocentric representations were identified by inclusively masking the [TTCallo-COLallo] contrast with the [TTCego-COLego] contrast (thresholded at $p<0.01$ uncorrected for multiple comparisons). This "conjunction" analysis defines a fundamental TTC network, which is recruited for TTC judgments whatever the viewpoint.

\section{Task $\times$ viewpoint interactions}

Regions preferentially activated by egocentric, rather than allocentric, representations of TTC were identified by the interaction between task and viewpoint. The interaction term allows for a comparison between egocentric and allocentric viewpoints, which would otherwise be subject to sensori-motor confounds due to the large differences in the visual display. However, by using the interaction term, the difference between TTC and colour judgements for egocentric displays is compared to the difference between TTC and colour judgements for allocentric displays, thus controlling for (or "subtracting out") more general, featureindependent task demands that are recruited by the TTC and colour tasks to the same degree. Due to the mathematical nature of the contrast, this interaction term will identify areas that are activated both by estimating TTC in egocentric displays (TTCego) and by estimating colour in allocentric displays (COLallo) ${ }^{1}$. Therefore, in order to pinpoint the areas that were recruited for egocentric TTC judgements, and not allocentric colour ones, we considered only those regions that were specific to the egocentric TTC condition (i.e we used the [TTCego-COLego] statistical map (defined above) as an inclusive mask, thresholded at $p<0.01$ uncorrected for multiple comparisons). We also examined the converse interaction ([TTCallo-COLallo] - [TTCego-COLego]) [inclusively masked by (TTCallo-COLallo, thresholded at $p<0.01$ uncorrected for multiple comparisons)] to examine areas specifically recruited for TTC judgements in allocentric displays.

\section{Parametric modulation of TTC-related activity by magnitude of tau-dot}

Regions associated more with contact than no-contact scenarios were identified by the parametric modulation of TTC regressors by the ranked tau-dot value per trial. The lowest tau-dot value (indexing a "sure-hit") was ranked 1 while the highest tau-dot value (indexing a "sure-miss") was ranked 16. The mid-range of tau-dot values indexed ambiguous trials for which it was more difficult to estimate whether the trial would result in contact or no-contact. Rank values were automatically mean-corrected by SPM software during the analysis. For both TTCego and TTCallo regressors, we identified areas whose activity varied as a linear or quadratic function of the ranked tau-dot value. For linear modulations, positive correlations indexed areas that responded more strongly to no-contact ("sure-miss") trials while negative correlations indexed areas that responded more strongly to contact ("sure-hit") trials. For quadratic modulations, positive correlations indexed areas that responded more strongly to obvious

${ }^{1}[$ TTCego-COLego $-[$ TTCallo-COLallo $]$ is mathematically equivalent to [TTCego-TTCallo] + [COLallo-COLego $]$. trials ("sure-hits" or "sure-misses"), while negative correlations indexed areas that responded more strongly to ambiguous trials. The SPM software automatically orthogonalised the quadratic modulation with respect to the linear one.

For each contrast of interest, all 12 maps (one per subject) were then entered into a second level of analysis. Statistical inferences for each contrast were derived using one-sample $t$-tests. The resulting activations were characterised in terms of both peak amplitude and spatial extent. The significance of each activation was estimated using distributional approximations from the theory of Gaussian fields. We adopted a significance threshold that was corrected for multiple comparisons (FDR corrected $p<0.05$ ), except in a priori regions of interest for which a more lenient threshold was used $(p<0.001$ uncorrected for multiple comparisons). Regions of interest were derived from existing fMRI literature on collision judgements and temporal expectancies (see Introduction) and comprised left-lateralised ventral premotor cortex, inferior parietal cortex and sensorimotor cortex. An uncorrected threshold $(p<0.001)$ was also used in the two analyses that examined change within a pre-defined network rather than the whole brain volume: (1) the interaction analyses, for which our masking procedure defined a posteriori a restricted search volume and (2) the parametric modulation analyses, which examined change in task-specific networks as a function of stimulus characteristics. All resulting $Z$-maps were additionally thresholded for cluster size at $p<0.05$.

\section{RESULTS}

\section{BEHAVIOURAL DATA}

There was no significant difference between TTC and COL tasks in terms of accuracy of performance $[F(1,11)=3.86$, ns $]$ or reaction time $[F(1,11)=3.60, \mathrm{~ns}]$, although there was a non-significant trend $(p=0.08)$ for TTC performance to be both less accurate and slower (Table 1 ). There was no significant difference between egocentric and allocentric viewpoints, as measured either by accuracy $[F(1,11)=3.35$, ns $]$ or RT $[F(1,11)=0.42, \mathrm{~ns}]$, nor a significant interaction between task and viewpoint, as measured either by accuracy $[F(1,11)=$ $2.65, \mathrm{~ns}]$ or RT $[F(1,11)=0.02, \mathrm{~ns}]$. These results indicate that the tasks were matched for overall difficulty.

These findings were confirmed by Signal Detection analysis (Table 1). Calculation of the sensitivity index, $d^{\prime}$ (a more sensitive measure of accuracy than \% correct that takes both hits and false alarms into account) for all four conditions revealed no significant main effect of task $[F(1,11)=0.73$, ns $]$, viewpoint $[F(1,11)=0.88, \mathrm{~ns}]$, nor interaction between task and viewpoint $[F(1,11)=1.26$, ns $]$. Calculation of response bias, $\beta$ (the likelihood of making a "yes or "no" response) revealed no significant difference between tasks $[F(1,11)=1.67$, ns $]$, but did identify a significant effect of viewpoint $[F(1,11)=45.08$, $p<0.001]$, that was qualified by a significant task $\times$ viewpoint interaction $[F(1,11)=12.77, p<0.005]$. Specifically, egocentric viewpoints provoked a more liberal response bias (more likely to say "yes") than allocentric ones, particularly in TTC tasks (Table 1). In other words, subjects were more likely to decide that a non-contact trial resulted in contact for egocentric trials than for allocentric ones.

We then examined performance as a function of the taudot value for each trial (Figure 2). Logistic regression analysis revealed a significant correlation between tau-dot value and the percentage of trials judged to result in contact (a "yes" decision) 
Table 1 | Mean (+SE) task performance during fMRI acquisition.

\begin{tabular}{lllll}
\hline & TTC allocentric & TTC egocentric & Colour allocentric & Colour egocentric \\
\hline Accuracy (\% correct) & $75.9(3.0)$ & $68.5(1.6)$ & $77.0(2.1)$ & $76.9(1.4)$ \\
Reaction time (ms) & $617.49(24.68)$ & $614.73(22.74)$ & $604.01(20.35)$ & $599.59(21.29)$ \\
$d^{\prime}$ & $1.62(0.18)$ & $1.40(0.11)$ & $1.65(0.15)$ & $1.68(0.11)$ \\
$\beta$ & $2.27(0.37)$ & $0.30(0.04)$ & $1.11(0.22)$ & $0.76(0.16)$ \\
\hline
\end{tabular}

Higher d' scores reflect more accurate performance. $A \beta$ score of 1 reflects a lack of response bias, lower $\beta$ scores reflect a more liberal response bias (more likely to say there was a collision/colour-match) while higher $\beta$ scores reflect a more conservative response bias (more likely to say there was no collision/colour-match).
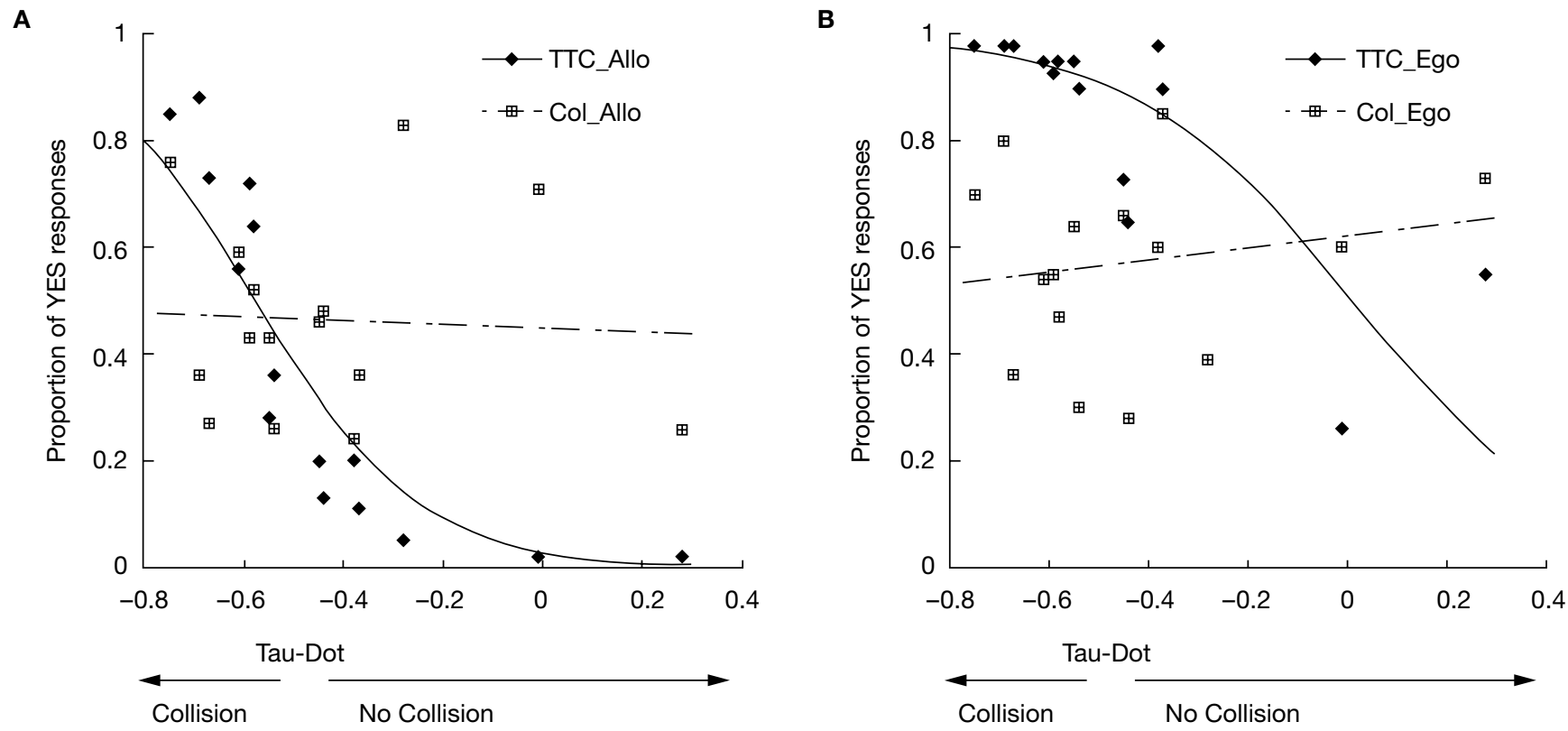

Figure 2 | Predictive value of tau-dot for making "contact" decisions. Mean performance (averaged across subjects) on TTC and colour tasks is plotted for each value of tau-dot for $(\boldsymbol{A})$ allocentric and $(\boldsymbol{B})$ egocentric viewpoints. Performance is measured as the proportion of trials in which the subject made a YES response (i.e. when the subject decided that a TTC trial would result in contact, or that a colour trial contained a colour-match). As expected, and for both alloentric and egocentric viewpoints, there was a significant relationship between tau-dot and percentage of trials judged to result in contact ( $\bullet$ ), but no significant relationship between tau-dot and the percentage of trials judged to result in a colour-match ( $(\boxplus)$. Specifically, the lower the tau-dot value the more likely the subject was to make a "contact" decision. Fitted curves represent the result of the logistic regression analysis. Each value of tau-dot was calculated using distances between the car and wall and the car's motion parameters (Supplementary Material 2). A tau-dot value of -0.5 represents the objective cut-off between a contact and no-contact trial.

for both the TTCego $\left[R^{2}(13)=0.60, p<0.001\right]$ and TTCallo $\left[R^{2}(13)=0.79, p<0.001\right]$ conditions. This indicates that the tau-dot value significantly predicted whether subjects would judge a particular scenario to result in contact or not. The lower the tau-dot value, the more likely subjects were to make a contact rather than no-contact decision. Conversely, there was no significant correlation between tau-dot value and the percentage of trials judged to result in a colour-match (a "yes" decision) for either the COLego $\left[R^{2}(13)=0.03, \mathrm{~ns}\right]$ or COLallo $\left[R^{2}(13)=0.002, \mathrm{~ns}\right]$ conditions. As expected, this suggests that tau-dot value had no predictive value for making colour judgements. Finally, in order to ensure that dynamic variables other than tau-dot could not also significantly predict TTC performance, we performed two additional logistic regression analyses between performance and (1) the car's final velocity and (2) the duration for which the animation was presented. Neither velocity nor duration significantly correlated with TTC performance in either the allocentric $\left[R^{2}(13)=0.29\right.$ and 0.16 , $\mathrm{ns}$, for velocity and duration respectively] or egocentric $\left[R^{2}(13)=0.13\right.$ and 0.05 , ns, for velocity and duration respectively] condition.

\section{fMRI DATA}

\section{Colour-related activity}

Egocentric representations of colour-processing activated visual area V1, visual area V4 bilaterally, intraparietal sulcus bilaterally and left middle frontal gyrus. Allocentric representations of colour-processing activated a more dorsal region of visual area V1, visual area V4 bilaterally, intraparietal sulcus bilaterally, left middle frontal gyrus and retrosplenial cortex (Table 2). Inclusive masking revealed that areas common to both egocentric and allocentric viewpoints were V4 bilaterally and intraparietal sulcus bilaterally (Figure 3A).

\section{TTC-related activity \\ Simple main effects and interactions}

Egocentric representations of TTC activated left ventral premotor cortex/frontal operculum, left inferior parietal cortex, and primary visual area V1. Allocentric representations of TTC activated a more distributed and bilateral network, including ventral premotor cortex/frontal operculum, medial rostral prefrontal cortex, inferior parietal cortex, dorsolateral posterior visual cortex, 
Table 2 | Colour network.

\begin{tabular}{|c|c|c|c|c|}
\hline \multirow[t]{2}{*}{ Anatomical structure } & \multicolumn{2}{|c|}{ Allocentric } & \multicolumn{2}{|c|}{ Egocentric } \\
\hline & $x, y, z(\mathrm{~mm})$ & $Z$ score & $x, y, z(\mathrm{~mm})$ & $Z$ score \\
\hline R ventrolateral visual cortex (V4) & $36,-87,-24$ & 4.50 & $30,-63,-15$ & 3.36 \\
\hline L ventrolateral visual cortex (V4) & $-36,-63,-24$ & 4.15 & $-27,-75,-24$ & 4.87 \\
\hline $\mathrm{R}$ intraparietal sulcus & $30,-66,42$ & 4.19 & $30,-66,42$ & 5.35 \\
\hline L intraparietal sulcus & $-30,-69,39$ & 3.65 & $-30,-72,33$ & 4.36 \\
\hline Left middle frontal gyrus & $-51,6,36$ & 4.30 & $-45,12,36$ & 4.07 \\
\hline Retrosplenial cortex & $6,-42,0$ & 4.75 & - & - \\
\hline Primary visual cortex (V1) & $0,-81,9$ & 3.69 & - & - \\
\hline
\end{tabular}

Areas of significantly increased regional activity during the colour minus TTC tasks for allocentric and egocentric viewpoints. Areas common to allocentric and egocentric viewpoints are illustrated in Figure 3A. $L=$ left, $R=$ right.

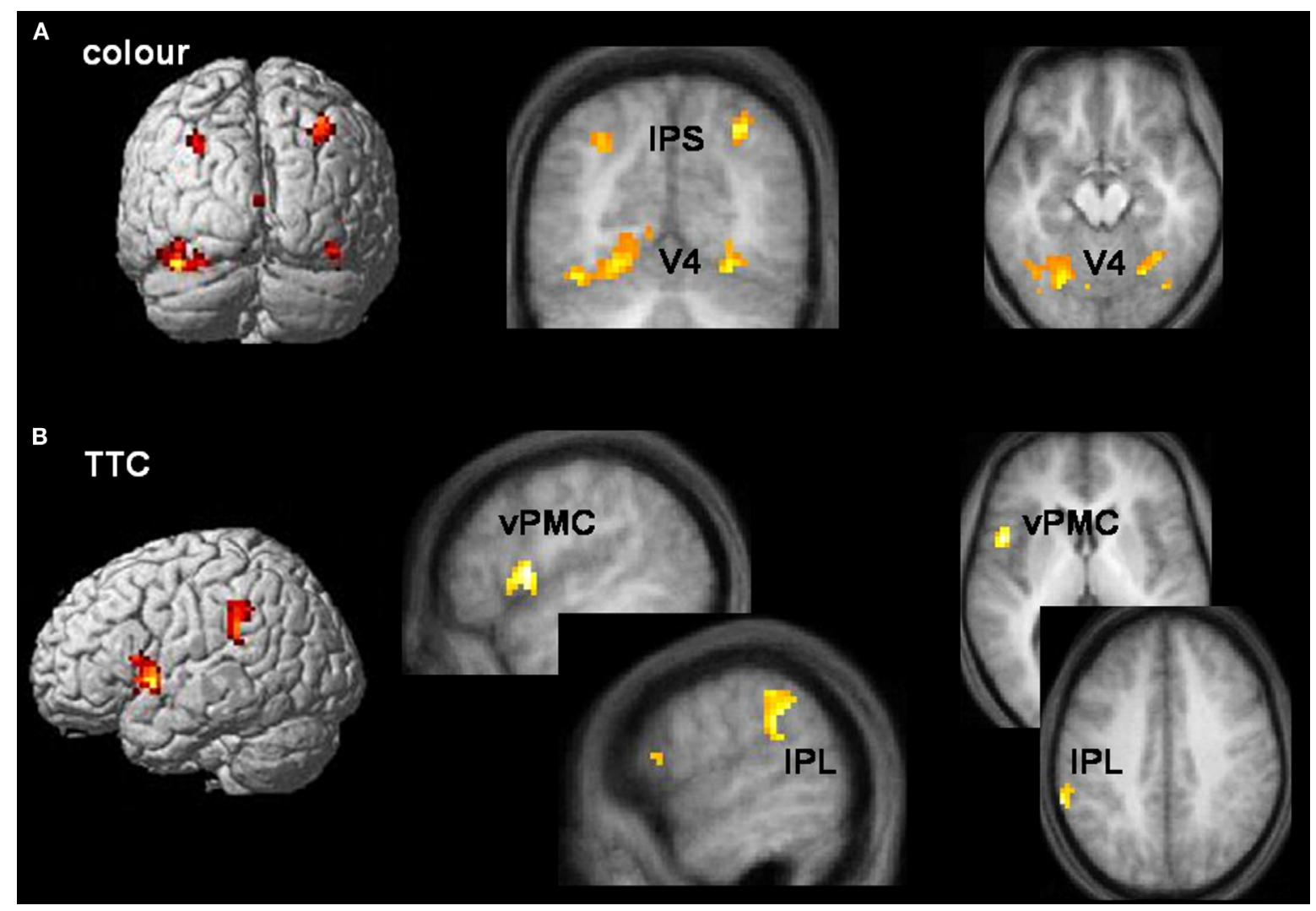

Figure 3 | Task-specific networks. (A) Colour tasks preferentially activated visual area V4 (x,y,z=-18,-75, -12; 36, $-60,-21)$ and intraparietal sulcus (IPS) $(x, y, z=-30,-69,39 ; 30,-66,42)$ bilaterally during both allocentric and egocentric viewpoints. Activations are rendered onto a standard template brain, and superimposed onto coronal $(y=-69 \mathrm{~mm})$ and transverse $(z=-18 \mathrm{~mm})$ slices of the averaged structural MRI. (B) TTC tasks preferentially activated left pars opercularis of the inferior frontal lobe (part of ventral premotor cortex (VPMC)) $(x, y, z=-51,6,3)$ and the supramarginal gyrus of left inferior parietal lobule $(\mathrm{PL})(x, y, z=-63,-45,39)$ during both allocentric and egocentric viewpoints. Activations are rendered onto a standard template brain, and superimposed onto saggital ( $x=-51$ and $-60 \mathrm{~mm}$ for vPMC and IPL activations respectively) and transverse ( $z=3$ and $39 \mathrm{~mm}$ for vPMC and IPL activations respectively) slices of the averaged structural MRI. Areas of activity common to allocentric and egocentric viewpoints were derived from a logical AND inclusive-masking procedure.

posterior cingulate and precuneus, as well as left-lateralised visual area V5 (also known as hMT+) (Table 3). Inclusive masking revealed that the only areas common to both egocentric and allocentric viewpoints were left pars opercularis of the inferior frontal lobe (BA44 and part of ventral premotor cortex) and the supramarginal gyrus of left inferior parietal cortex (BA40) (Figure 3B). Interaction analyses revealed that the only region selectively activated by egocentric, rather than allocentric, representations of TTC was primary visual area $\mathrm{V} 1(x, y, z$ co-ordinates $=-6,-105$, $6 \mathrm{~mm}$ ) while the only region selectively activated by allocentric, rather than egocentric, representations of TTC was left-lateralised visual area V5 $(x, y, z$ co-ordinates $=-54,-69,3 \mathrm{~mm})$.

\section{Modulation of TTC-related activity by magnitude of tau-dot}

The logistic regression analysis confirmed that tau-dot was a good predictor of TTC judgements, particularly for the allocentric viewpoint. We therefore examined how the magnitude of tau-dot modulated TTC-related activity from both egocentric and allocentric viewpoints. We modelled both linear and quadratic modulations of neural activity as a function of ranked tau-dot value. 
Table 3 | TTC network.

\begin{tabular}{|c|c|c|c|c|}
\hline \multirow[t]{2}{*}{ Anatomical structure } & \multicolumn{2}{|c|}{ Allocentric } & \multicolumn{2}{|c|}{ Egocentric } \\
\hline & $x, y, z(\mathrm{~mm})$ & $Z$ score & $x, y, z(\mathrm{~mm})$ & $Z$ score \\
\hline L ventral premotor cortex/frontal operculum (BA44) & $-51,6,3$ & 4.46 & $-45,3,0$ & 3.93 \\
\hline L inferior parietal cortex (BA40) & $-63,-45,39$ & 4.32 & $-57,-45,39$ & 3.66 \\
\hline R rostral prefrontal cortex (BA10) & $12,60,-6$ & 3.98 & - & - \\
\hline $\mathrm{R}$ posterior cingulate/precuneus & $9,-42,42$ & 4.93 & - & - \\
\hline L posterior cingulate /precuneus & $-15,-30,42$ & 4.37 & - & - \\
\hline $\mathrm{R}$ dorsolateral visual cortex (BA19) & $42,-84,27$ & 3.99 & - & - \\
\hline L dorsolateral visual cortex (BA19) & $-45,-75,24$ & 4.22 & - & - \\
\hline L lateral visual cortex (V5) & $-57,-72,9$ & 3.97 & _ & - \\
\hline Primary visual cortex (V1) & - & - & $-6,-105,3$ & 4.36 \\
\hline
\end{tabular}

Areas of significantly increased regional activity during the TTC minus colour tasks for allocentric and egocentric viewpoints. Areas common to allocentric and egocentric viewpoints are illustrated in Figure 3B. $L=$ left, $R=$ right, $B A=$ Brodmann's area.

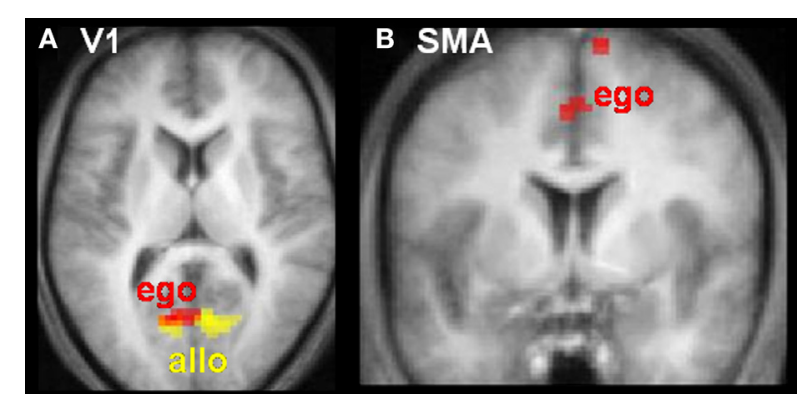

Figure 4 | Neural activity varies as a function of tau-dot. Activity in (A) primary visual cortex (V1) and (B) Supplementary Motor Area (SMA) increased as tau-dot value decreased, indicating a parametric response to the increasing certitude of a collision (see Figure 2 for the behavioural corollary of this response). V1 activity was modulated during both allocentric (yellow) and egocentric (red) viewpoints, whereas SMA activity was modulated during egocentric viewpoints only. Activations are displayed on transverse $(z=9 \mathrm{~mm})$ and coronal $(y=0 \mathrm{~mm})$ slices of the averaged structural MRI of all 12 subjects.

Linear modulations indexed the likelihood of making a contact versus no-contact judgement. The lower the tau-dot value (i.e. the more likely to be associated with a contact judgement) the more activity increased in visual area V1/2 for both egocentric and allocentric viewpoints (Figure 4A), and additionally in SMA for egocentric viewpoints only (Figure 4B). The higher the taudot value (i.e. the more likely to be associated with a no-contact judgement), the more activity increased in anterior cingulate for both egocentric and allocentric viewpoints, and additionally in left IPS, bilateral inferior frontal cortex and left ventromedial visual cortex for egocentric viewpoints only (Table 4).

Quadratic modulations indexed the certainty of the scenario's outcome, regardless of whether it was a contact or no-contact trial. The more intermediate the tau-dot value (i.e. indexing a more "ambiguous" trial and thus more likely to be associated with an incorrect response), the more activity increased in right temporal pole for allocentric viewpoints and in mid/superior temporal cortex bilaterally, left inferior parietal cortex and dorsomedial visual cortex for egocentric viewpoints (Table 4). There was no significant modulation of activity as the tau-dot value became more extreme (i.e. indexing a more "obvious" trial and thus more likely to be associated with a correct response).

\section{DISCUSSION}

Estimating the time-to-contact (TTC) of approaching objects is critical for formulating future action plans. We used an ecologically valid driving simulation, in which a braking car approached a distant wall, to index the use of TTC information in assessing the potential for collision. This task did not require an explicit temporal estimate of TTC but, rather, a prediction of what would happen at a specific moment in time. Event-related fMRI data showed selective activation of left pars opercularis of the inferior frontal lobe (part of ventral premotor cortex, and also known as frontal operculum) and the supramarginal gyrus of left inferior parietal cortex during TTC tasks (Figure 3B). We confirmed the functional specificity of these findings by comparing TTC tasks to colour controls that were matched for both visuo-motor task demands and task difficulty. We also confirmed the visuo-spatial generality of the TTC-induced activity by demonstrating activation of this network whether the subject was viewing the approach from an egocentric (driver's viewpoint) or allocentric (bird's-eye view) viewpoint. However, we also found differences between these two conditions such that allocentric judgements selectively activated visual area V5 (hMT+), while egocentric TTC judgements selectively activated primary visual cortex, area V1. V1 activity also varied as a function of the increasing certainty of potential collision. Finally, there was notable overlap between the leftsided premotor-parietal regions activated by the TTC task and those previously implicated in a temporal attentional orienting task (Coull and Nobre, 1998). Despite widely-differing visual and task demands, both paradigms encourage subjects to use predictive information (stimulus motion or attentional cues) to project forwards to a precise moment in time. Based on the anatomical and functional correspondence between these two paradigms, we therefore suggest that left ventral premotor and parietal cortices are critically involved in temporal prediction.

\section{GENERALITY OF THE TEMPORAL PREDICTION NETWORK}

Maintaining a constant interval ("foreperiod") between warning and target stimuli across trials allows temporal regularities to be implicitly learned over time, so encouraging temporal prediction and, thus, speeding responses to target stimuli (Niemi and Näätänen, 1981). Fixed, compared to random, foreperiods activate left premotor cortex, left intra-parietal sulcus and preSMA (Sakai et al., 2000). Left ventral premotor and parietal areas are also activated by temporally predictable stimuli in a temporal attentional orienting task (Coull and Nobre, 1998; Coull et al., 2000, 2001), in which temporally-predictive information is conveyed explicitly by an attentional cue, rather than being implicitly picked-up over numerous stimulus repetitions. A very 


\begin{tabular}{|c|c|c|c|c|}
\hline \multirow[t]{2}{*}{ Anatomical structure } & \multicolumn{2}{|c|}{ Allocentric } & \multicolumn{2}{|c|}{ Egocentric } \\
\hline & $x, y, z(\mathrm{~mm})$ & $Z$ score & $x, y, z(\mathrm{~mm})$ & $Z$ score \\
\hline \multicolumn{5}{|l|}{ LINEAR MODULATIONS } \\
\hline \multicolumn{5}{|l|}{ Positively correlated with tau-dot } \\
\hline $\mathrm{R}$ anterior cingulate & $9,30,15$ & 3.66 & $-9,30,33$ & 4.43 \\
\hline R inferior frontal cortex (BA44) & - & - & $48,3,15$ & 3.86 \\
\hline L inferior frontal cortex (BA45) & - & - & $-48,24,15$ & 3.80 \\
\hline L intraparietal sulcus & - & - & $-33,-60,24$ & 3.81 \\
\hline L ventromedial visual cortex & - & - & $-21,-99,-9$ & 4.51 \\
\hline \multicolumn{5}{|l|}{ Negatively correlated with tau-dot } \\
\hline Primary visual cortex (V1) & $-15,-75,12$ & 4.66 & $-6,-72,9$ & 4.54 \\
\hline SMA - ventral & - & - & $-3,3,48$ & 4.16 \\
\hline SMA - dorsal & & - & $12,0,75$ & 3.96 \\
\hline \multicolumn{5}{|l|}{ QUADRATIC MODULATIONS } \\
\hline \multicolumn{5}{|l|}{ Negatively correlated with tau-dot } \\
\hline R temporal pole (BA22) & $60,-9,6$ & 4.81 & - & - \\
\hline L inferior parietal cortex (BA40) & - & - & $-51,-57,39$ & 3.36 \\
\hline R superior temporal gyrus (BA22) & - & - & $57,-57,21$ & 4.19 \\
\hline L middle temporal gyrus (BA21) & - & - & $-60,-48,-6$ & 3.91 \\
\hline $\mathrm{R}$ dorsomedial visual cortex & - & - & $15,-90,30$ & 3.67 \\
\hline
\end{tabular}

Linear and quadratic modulations of TTC-specific activity as a function of tau-dot. Data are reported for both allocentric and egocentric viewpoints. $L=$ left, $R=$ right. BA $=$ Brodmann's area.

similar left-lateralised network is also activated by TTC judgements in the current experiment, in which spatio-temporallypredictive information was conveyed implicitly (this time on a trial-by-trial basis) by the trajectory of the moving car. Moreover, previous fMRI studies of TTC tasks have also shown activation of left-lateralised inferior parietal and ventral premotor areas (Assmus et al., 2003, 2005). Activation of similar cortical networks by these three distinct paradigms suggests a common underlying system for temporal prediction, regardless of whether explicit, cognitively-controlled, top-down processing (temporal orienting), or implicit, stimulus-driven, bottom-up processing (fixed-foreperiod or TTC) was used to make the prediction.

The TTC task differs from the colour control task on processes other than temporal prediction (for example, attention to motion, spatio-temporal integration or even more emotional factors, such as risk assessment). One may therefore argue that the left premotor and parietal activations actually index these visual or emotional aspects of the task. However, we believe they are more likely to represent temporal prediction based on the anatomical and functional similarity of the TTC task with the temporal orienting task, which contains a strong element of temporal prediction but not of motion processing, spatio-temporal integration nor risk assessment. It may also be argued that the left-lateralisation of the temporal prediction network in the current study is due to the fact that subjects always responded with their right hand. However, right-hand responses were also required for the colour task to which the TTC task was directly compared, making this an unlikely explanation.

Intriguingly, a recent fMRI study that also used spatiotemporal trajectories to measure perceptual prediction ( $O$ 'Reilly et al., 2008) found a right-lateralised parietal-premotor network for spatio-temporal (velocity) judgements as opposed to spatial (direction) ones. They suggested that lateralisation differences between their study (right-sided) and previous temporal orienting studies (left-sided) could be due to the greater spatial or perceptual demands of their task. However, spatio-temporal trajectories and perceptual discrimination tasks were also used in the current study, as well as in studies by Assmus et al. (2003, 2005), and both groups found left-lateralised activations. The study by O'Reilly et al. (2008) also observed selective activation in cerebellum for spatio-temporal prediction, which we did not observe in the current study. We did not scan the ventral third of the cerebellum, which could have contributed to the lack of effect. However, an alternative explanation is that O'Reilly et al. (2008) examined temporal prediction over a short duration $(600 \mathrm{~ms})$ whereas our own study and that of Assmus et al. $(2003,2005)$ examined temporal prediction over longer durations $(\sim 1.5-3.5 \mathrm{~s})$. Indeed, recent transcranial magnetic stimulation (TMS) evidence (Koch et al., 2007; Lee et al., 2007) suggests that the cerebellum is particularly implicated in the timing of short (millisecond) durations.

In the temporal orienting task, temporal prediction is measured by the speed with which a motor response is executed after a predictable delay. In the TTC paradigm however, temporal prediction is measured by the accuracy with which subjects can predict a potential collision between two perceptual objects. Despite its reliance on perceptual discrimination (see also the perceptual tasks used by Assmus et al., 2003, 2005), the TTC task activated regions of premotor and parietal cortices similar to those previously activated by the temporal orienting speeded RT task. Schubotz (2007) has recently proposed that the same neural circuitry (areas traditionally implicated in forward models of motor processing) can be used to make both motor (actions) and perceptual (events) predictions. Our findings are concordant with this action-perception overlap and, furthermore, agree with specific findings by Schubotz et al. (2003) that ventral premotor cortex is preferentially engaged when making temporal (as opposed to spatial or object-based) predictions in a serial prediction task (Schubotz and von Cramon, 2001; Schubotz et al., 2003). Schubotz's (2007) HAPEM (Habitual Pragmatic Event Map) model states that event prediction within a particular domain (e.g. spatial or temporal) engages activity in 
the effector-specific motor circuit that is most suited to process that domain. For example, spatially-predictable events activate visual saccade motor circuits, whereas temporally-predictable events activate more ventral speech circuits (Wolfensteller et al., 2007). Our own observation that TTC estimation activates the pars opercularis of the left inferior frontal lobe, which comprises part of Broca's area, and the supramarginal gyrus of inferior parietal cortex, which is implicated in verbal working memory (Muller and Knight, 2006; Smith and Jonides, 1998), further corroborates this model. Furthermore, we suggest that co-activation of pars opercularis and supramarginal gyrus actually reflects the use of a learned temporal stimulus structure rather than more general processes of language (Hammond, 1982; Merzenich et al., 1996; Tallal et al., 1993) or prediction since temporally-structured stimuli are common to both speech comprehension and temporal prediction (both of which activate this cortical network) whereas language or (non-temporal) prediction are not necessarily common to both.

\section{TTC ESTIMATION DURING HEAD-ON VERSUS LATERAL APPROACHES}

The original formulation of tau theory (Lee, 1976) stated that tau, a visual feature extracted directly from the optic flow, could be used to estimate TTC without recourse to complex calculations involving distance and velocity. Later critics suggested that although tau may be involved in TTC estimation, it is not the only variable used (Tresilian, 1999). In our tasks, subjects made use of TTC information to estimate whether a braking car would eventually collide with a distant wall. The activation of a left fronto-parietal network in the present study indicates that, at least for our tasks, subjects did not rely solely on optical information but also engaged more cognitive or attentional strategies (e.g. temporal prediction, spatio-temporal integration etc.). Furthermore, despite clear differences in visual input, this network was activated both by head-on (egocentric) and lateral (allocentric) approaches, suggesting a common functional mechanism. As outlined above, we believe this cognitive mechanism to be temporal prediction.

A more recent formulation of tau theory suggests that while TTC in head-on approaches may be estimated using the rate of image dilation, TTC in lateral approaches relies on the rate of closure of the gap between the car and the wall (Lee, 1998). When we compared egocentric TTC tasks to allocentric ones directly we observed differences in (bottom-up) early visual processing to parallel these functional differences. Egocentric TTC trials selectively activated primary visual cortex (V1), whereas allocentric ones selectively activated visual area V5/hMT+. These visual activations cannot entirely be explained by basic visual processing of optic flow (e.g. Wunderlich et al., 2002) or lateral translations (e.g. Cheng et al., 1995) respectively, since each TTC task was first compared to a control task that employed exactly the same visual stimuli in a fully-factorial design. Note also, that this design would control for possible activation of other cortical areas previously associated with processing of optic flow such as V5/hMT+ complex (Morrone et al., 2000; Smith et al., 2006) or intraparietal sulcus (Bremmer et al., 2002; Wall and Smith, 2008). Rather, differential activation of V1 and V5 during the direct comparison of egocentric and allocentric tasks is most likely to reflect top-down attentional modulation of feature-specific visual processing areas (Corbetta et al., 1990; Gandhi et al., 1999; Martinez et al., 1999; O'Craven et al., 1997; Somers et al., 1999). Specifically, we suggest modulation of V1 during egocentric tasks reflects the fact that TTC information was estimated by attending to the rate of image dilation derived from the optic flow of a head-on approach. On the other hand, modulation of V5/MT during allocentric tasks reflects estimation of TTC information by attention to the rate of gap closure derived from the lateral visual translations of a transversal approach. These neural differences suggest that using TTC information derived from image dilation or gap closure to predict the potential for collision does not depend upon the same visual mechanisms although, as proposed earlier, similar temporal prediction strategies (underpinned by the left premotor-parietal network) may be involved in both. Indirect anatomical connections between visual and parietal-premotor areas (Andersen et al., 1990; Felleman and Van Essen, 1991; Ungerleider and Desimone, 1986) would allow visual TTC information to be incorporated into the temporal prediction network. Finally, note that although eye movement patterns could be hypothesised to differ across each of the four conditions (unfortunately we could not measure eye movements during scanning), there was no evidence of preferential frontal eye-field activation for one condition compared to another. Thus, potential eye movement confounds are unlikely to significantly contribute to these results.

However, V1 activity also varied inversely as a function of the value of tau-dot for both egocentric and allocentric viewpoints. Specifically, V1 activity was higher when the trial specified a surehit rather than a sure-miss. Therefore, V1 responded preferentially to potential collisions, whatever the viewpoint. Since more trials were judged to result in contact during egocentric trials (i.e. increased false alarms) than allocentric ones, the aforementioned increase in V1 activity during egocentric trials may not only reflect attentional modulation of optic flow but may also reflect the increased incidence of subjective collisions during these trials. In other words, the increasing attentional salience of an increasingly certain collision would modulate activity in primary visual cortex, whatever the viewpoint. Indeed, it makes evolutionary sense that such a biologically-critical function (collision judgements) would modulate activity in such early visual processing areas (see also Wunderlich et al., 2002).

\section{MOTOR PREPARATION DURING THE PERCEPTION OF HEAD-ON COLLISIONS}

Behavioural data were extremely similar to previous psychophysical examinations of allocentric and egocentric TTC tasks (Bootsma and Craig, 2003), both in terms of performance accuracy and the predictive value of tau-dot. The objective value of tau-dot on any given trial was a good predictor of subjective perception, such that lower tau-dot values corresponded to an increased likelihood of making a contact judgement (sure-hits), while higher tau-dot values corresponded to increased likelihood of making a no-contact judgement (sure-misses). Signal Detection analysis revealed that egocentric tasks elicited a significantly higher incidence of false alarms than allocentric ones, such that subjects were more likely to make "contact" decisions, even for non-contact trials (Figure 2). This relatively liberal response bias for egocentric versus allocentric trials, also observed by Bootsma and Craig (2003), may indicate increased caution for head-on approaches that would allow situations potentially harmful to the observer to be avoided. Increased caution may be implemented either by simply categorising all ambiguous trials as collision trials in a "better safe than sorry" approach and/or by subjectively shifting the point of potential contact forward to a location somewhat in front of the actual point of contact (i.e. to 
a point somewhere in front of the car's bonnet) so as to provide a larger safety margin.

Intriguingly, SMA activity varied inversely as a function of taudot for egocentric, but not allocentric, viewpoints. Specifically, SMA activity was higher for sure-hits than sure-misses. Given SMA's central role in motor preparation (e.g. Lee et al., 1999) we hypothesise that, during head-on approaches, subjects activated the neural representation of motor avoidance mechanisms, despite the fact that a passive perceptual judgement was all that was required (see also Schubotz, 2007). These increases in SMA activity may therefore reflect a neural defence strategy deployed in response to the increased behavioural caution that is observed in egocentric trials.

\section{CONCLUSIONS}

During the TTC task, subjects predicted whether a braking car would eventually collide with a distant wall. This task did not require an explicit temporal estimate of TTC but, rather, a prediction of what would happen at a specific moment in time. Given the neural overlap with previous studies of temporal orienting, our results support the existence of a core network for temporal prediction in the pars opercularis of left inferior frontal lobe (part of ventral premotor cortex) and the supramarginal gyrus of left inerior parietal cortex. We further suggest that these results may index the activation of a more fundamental functional circuit that represents temporal stimulus structure. We also observed differential activation of visual areas V1 and $\mathrm{V} 5$ by egocentric and allocentric viewpoints respectively, which most likely reflects top-down attentional modulation of featurespecific visual processing areas for optic-flow and lateral motion. Taken together, these results suggest that collision judgements engage task-independent representations of temporal prediction as well as modulating activity at a local level in functionally specialised (visual) areas. Finally, we demonstrated that the optical variable tau-dot held predictive value for making collision judgements and that the increasing certitude of a collision modulated activity in V1, whatever the viewpoint. That collision certitude modulated such an early stage of visual processing may reflect the biological importance, and consequent attentional saliency, of potential collisions.

\section{CONFLICT OF INTEREST STATEMENT}

The authors declare that the research was conducted in the absence of any commercial or financial relationships that could be construed as a potential conflict of interest.

\section{ACKNOWLEDGEMENTS}

With thanks to Muriel Roth and Jean-Luc Anton for scanning support. This work was funded by a Marie Curie Fellowship of the European Community programme Human Potential, under contract number HPMF-CT-2002-01525.

\section{SUPPLEMENTARY MATERIAL 1}

A supplementary movie file can be found online at: http:// frontiersin.org/humanneuroscience/paper/10.3389/neuro.09/ 010.2008/.

The movie shows four typical trials, presented in the order TTCallo, COLego, CLOallo, TTCego. The subject's task was to make a decision (yes/no) based on potential contact (TTC tasks) or colour-match (COL tasks) between the car and the wall. For example, in the TTCallo trial, the car would have come into contact with the wall had it continued its trajectory therefore the correct response is Yes ("oui" in french) requiring a middle finger response (corresponding to the right-sided location of "oui" on the response screen). In the COLego task there is no colour-match between the car and the wall therefore the correct response is No ("non" in french) requiring an index finger response (corresponding to the left-sided location of "non" on the response screen). These movie files $(25 \mathrm{~Hz})$ were created for the purposes of illustration only and are of lower resolution than the actual stimulus display $(60 \mathrm{~Hz})$ that was shown to subjects.

\section{SUPPLEMENTARY MATERIAL 2}

See Table S1

Table S1 | The 16 different values of tau-dot at animation offset were calculated using the intial distance of the car from the wall, the car's initial velocity, the car's rate of deceleration, and the final distance from the wall when the animation ended. This resulted in eight scenarios in which there would have been contact between the car and wall had the animation continued (tau-dot $<-0.5$ ), and eight in which there would have been no contact (taudot $\geq-0.5$ ). At animation onset, tau-dot values varied around -0.5 for all scenarios (range from -0.447 to -0.548 ) meaning subjects could not yet accurately predict whether or not there would be a collision. Note that tau-dot is orthogonal to speed or image size since different tau-dot values result from different combinations of motion or distance variables.

\begin{tabular}{|c|c|c|c|c|c|c|}
\hline Initial distance (m) & Initial velocity $\left(\mathbf{m}^{-1}\right)$ & Deceleration $\left(\mathrm{m}^{-2}\right)$ & Final distance $(\mathrm{m})$ & Contact & Display time (s) & Tau-dot \\
\hline 57.69 & 30.3 & 7.2 & 6 & Yes & 2.38 & -0.75 \\
\hline 57.69 & 29.7 & 7.2 & 6 & Yes & 2.49 & -0.69 \\
\hline 57.69 & 30.3 & 7.2 & 12 & Yes & 1.97 & -0.67 \\
\hline 57.69 & 29.7 & 7.2 & 12 & Yes & 2.05 & -0.61 \\
\hline 62.505 & 30.3 & 7.2 & 6 & Yes & 2.79 & -0.59 \\
\hline 57.69 & 30.3 & 7.8 & 6 & Yes & 2.53 & -0.58 \\
\hline 62.505 & 30.3 & 7.2 & 12 & Yes & 2.29 & -0.55 \\
\hline 57.69 & 30.3 & 7.8 & 12 & Yes & 2.05 & -0.54 \\
\hline 57.69 & 29.7 & 7.8 & 12 & No & 2.14 & -0.45 \\
\hline 62.505 & 29.7 & 7.2 & 12 & No & 2.40 & -0.44 \\
\hline 57.69 & 29.7 & 7.8 & 6 & No & 2.69 & -0.38 \\
\hline 62.505 & 29.7 & 7.2 & 6 & No & 2.98 & -0.37 \\
\hline 62.505 & 30.3 & 7.8 & 12 & No & 2.42 & -0.28 \\
\hline 62.505 & 29.7 & 7.8 & 12 & No & 2.56 & -0.01 \\
\hline 62.505 & 30.3 & 7.8 & 6 & No & 3.11 & 0.28 \\
\hline 62.505 & 29.7 & 7.8 & 6 & No & 3.71 & 75.47 \\
\hline
\end{tabular}




\section{REFERENCES}

Andersen, R. A., Asanuma, C., Essick, G., and Siegel, R. M. (1990). Corticocortical connections of anatomically and physiologically defined subdivisions within the inferior parietal lobule. J. Comp. Neurol. 296, 65-113.

Assmus, A., Marshall, J. C., Noth, J., Zilles, K., and Fink, G. R. (2005). Difficulty of perceptual spatiotemporal integration modulates the neural activity of left inferior parietal cortex. Neuroscience 132, 923-927.

Assmus, A., Marshall, J. C., Ritzl, A., Noth, J., Zilles, K., and Fink, G. R. (2003). Left inferior parietal cortex integrates time and space during collision judgments. Neuroimage 20(Suppl. 1), S82-S88.

Bootsma, R. J., and Craig, C. M. (2003). Information used in detecting upcoming collision. Perception 32, 525-544.

Bremmer, F., Duhamel, J. R., Ben Hamed, S., and Graf, W. (2002). Heading encoding in the macaque ventral intraparietal area (VIP). Eur. J. Neurosci. 16, 1554-1568

Cheng, K., Fujita, H., Kanno, I., Miura, S., and Tanaka, K. (1995). Human cortical regions activated by wide-field visual motion: an H2(15)O PET study. J. Neurophysiol. 74, 413-427.

Corbetta, M., Miezin, F. M., Dobmeyer, S., Shulman, G. L., and Petersen, S. E. (1990). Attentional modulation of neural processing of shape, color, and velocity in humans. Science 248, 1556-1559.

Coull, J. T., Frith, C. D., Buchel, C., and Nobre, A. C. (2000). Orienting attention in time: behavioural and neuroanatomical distinction between exogenous and endogenous shifts. Neuropsychologia 38, 808-819.

Coull, J. T., and Nobre, A. C. (1998). Where and when to pay attention: the neural systems for directing attention to spatial locations and to time intervals as revealed by both PET and fMRI. J. Neurosci. 18, 7426-7435.

Coull, J. T., Nobre, A. C., and Frith, C. D. (2001). The noradrenergic alpha2 agonist clonidine modulates behavioural and neuroanatomical correlates of human attentional orienting and alerting. Cereb. Cortex 11, 73-84.

Coull, J. T., Vidal, F., Nazarian, B., and Macar, F. (2004). Functional anatomy of the attentional modulation of time estimation. Science 303, 1506-1508.

Felleman, D. J., and Van Essen, D. C. (1991). Distributed hierarchical processing in the primate cerebral cortex. Cereb. Cortex 1, 1-47.

Field, D. T., and Wann, J. P. (2005). Perceiving time to collision activates the sensorimotor cortex. Curr. Biol. 15, 453-458.

Friston, K. J., Fletcher, P., Josephs, O., Holmes, A., Rugg, M. D., and Turner, R. (1998). Event-related fMRI: characterizing differential responses. Neuroimage 7, 30-40.

Friston, K. J., Frith, C. D., Turner, R., and Frackowiak, R. S. (1995a). Characterizing evoked hemodynamics with fMRI. Neuroimage 2, 157-165.

Friston, K. J., Holmes, A. P., Poline, J. B., Grasby, P. J., Williams, S. C., Frackowiak, R. S., and Turner, R. (1995b). Analysis of fMRI time-series revisited. Neuroimage 2, 45-53.

Gandhi, S. P., Heeger, D. J., and Boynton, G. M. (1999). Spatial attention affects brain activity in human primary visual cortex. Proc. Natl. Acad. Sci. USA 96, 3314-3319.

Hammond, G. R. (1982). Hemispheric differences in temporal resolution. Brain Cogn. 1, 95-118.

Josephs, O., and Henson, R. N. (1999). Event-related functional magnetic resonance imaging: modelling, inference and optimization. Philos. Trans. R. Soc Lond. B Biol. Sci. 354, 1215-1228.

Kim, N. G., Turvey, M. T., and Carello, C. (1993). Optical information about the severity of upcoming contacts. J. Exp. Psychol. Hum. Percept. Perform. 19, 179-193.

Koch, G., Oliveri, M., Torriero, S., Salerno, S., Lo Gerfo, E., and Caltagirone, C (2007). Repetitive TMS of cerebellum interferes with millisecond time processing. Exp. Brain Res. 179, 291-299.

Lee, D. (1998). Guiding movement by coupling taus. Ecol. Psychol. 10, 221-250.

Lee, D. N. (1976). A theory of visual control of braking based on information about time-to-collision. Perception 5, 437-459.

Lee, K.-H., Egleston, P. N., Brown, W. H., Gregory, A. N., Barker, A. T., and Woodruff, P. W. R. (2007). The role of the cerebellum in subsecond time perception: evidence from repetitive transcranial magnetic stimulation. J. Cogn Neurosci. 19, 147-157.
Lee, K. M., Chang, K. H., and Roh, J. K. (1999). Subregions within the supplementary motor area activated at different stages of movement preparation and execution. Neuroimage 9, 117-123.

Martinez, A., Anllo-Vento, L., Sereno, M. I., Frank, L. R., Buxton, R. B. Dubowitz, D. J., Wong, E. C., Hinrichs, H., Heinze, H. J., and Hillyard, S. A. (1999). Involvement of striate and extrastriate visual cortical areas in spatial attention. Nat. Neurosci. 2, 364-369.

Merzenich, M. M., Jenkins, W. M., Johnston, P., Schreiner, C., Miller, S. L., and Tallal, P. (1996). Temporal processing deficits of language-learning impaired children ameliorated by training. Science $271,77-81$.

Morrone, M. C., Tosetti, M., Montanaro, D., Fiorentini, A., Cioni, G., and Burr, D. C. (2000). A cortical area that responds specifically to optic flow, revealed by fMRI. Nat. Neurosci. 3, 1322-1328.

Muller, N. G., and Knight, R. T. (2006). The functional neuroanatomy of working memory: contributions of human brain lesion studies. Neuroscience 139, 51-58.

Niemi, P., and Näätänen, R. (1981). Foreperiod and simple reaction time. Psychol. Bull. 89, 133-162.

Nobre, A. C. (2001). Orienting attention to instants in time. Neuropsychologia 39, 1317-1328.

O'Craven, K. M., Rosen, B. R., Kwong, K. K., Treisman, A., and Savoy, R. L. (1997). Voluntary attention modulates fMRI activity in human MT-MST. Neuron 18 , 591-598.

O'Reilly, J. X., Mesulam, M. M., and Nobre, A. C. (2008). The cerebellum predicts the timing of perceptual events. J. Neurosci. 28, 2252-2260.

Rock, P. B., and Harris, M. G. (2006). Tau as a potential control variable for visually guided braking. J. Exp. Psychol. Hum. Percept. Perform. 32, 251-267.

Rushworth, M. F., Johansen-Berg, H., Gobel, S. M., and Devlin, J. T. (2003). The left parietal and premotor cortices: motor attention and selection. Neuroimage 20(Suppl. 1), S89-S100.

Sakai, K., Hikosaka, O., Takino, R., Miyauchi, S., Nielsen, M., and Tamada, T. (2000). What and when: parallel and convergent processing in motor control. J. Neurosci. 20, 2691-2700.

Schubotz, R. I. (2007). Prediction of external events with our motor system: towards a new framework. Trends Cogn. Sci. 11, 211-218.

Schubotz, R. I., and von Cramon, D. Y. (2001). Functional organization of the lateral premotor cortex: fMRI reveals different regions activated by anticipation of object properties, location and speed. Brain Res. Cogn. Brain Res. 11, 97-112.

Schubotz, R. I., von Cramon, D.Y., and Lohmann, G. (2003). Auditory what, where, and when: a sensory somatotopy in lateral premotor cortex. Neuroimage 20, $173-185$.

Smith, A. T., Wall, M. B., Williams, A. L., and Singh, K. D. (2006). Sensitivity to optic flow in human cortical areas MT and MST. Eur. J. Neurosci. 23, 561-569.

Smith, E. E., and Jonides, J. (1998). Neuroimaging analyses of human working memory. Proc. Natl. Acad. Sci. USA 95, 12061-12068.

Somers, D. C., Dale, A. M., Seiffert, A. E., and Tootell, R. B. (1999). Functional MRI reveals spatially specific attentional modulation in human primary visual cortex. Proc. Natl. Acad. Sci. USA 96, 1663-1668.

Tallal, P., Miller, S., and Fitch, R. H. (1993). Neurobiological basis of speech: a case for the preeminence of temporal processing. Ann. NY Acad. Sci. 682, 27-47.

Tresilian, J. R. (1999). Visually timed action: time-out for 'tau'? Trends Cogn. Sci. $3,301-310$.

Ungerleider, L. G., and Desimone, R. (1986). Cortical connections of visual area MT in the macaque. J. Comp. Neurol. 248, 190-222.

Wall, M. B., and Smith, A. T. (2008). The representation of egomotion in the human brain. Curr. Biol. 18, 191-194.

Wolfensteller, U., Schubotz, R. I., and von Cramon, D. Y. (2007). Understanding non-biological dynamics with your own premotor system. Neuroimage 36(Suppl. 2), T33-T43.

Wunderlich, G., Marshall, J. C., Amunts, K., Weiss, P. H., Mohlberg, H., Zafiris, O., Zilles, K., and Fink, G. R. (2002). The importance of seeing it coming: a functional magnetic resonance imaging study of motion-in-depth towards the human observer. Neuroscience 112, 535-540.

Yilmaz, E. H., and Warren, W. H., Jr (1995). Visual control of braking: a test of the tau hypothesis. J. Exp. Psychol. Hum. Percept. Perform. 21, 996-1014. 